\title{
Coeficientes Convectivos de Transferencia de Calor durante el Freído de Láminas de Tilapia (Oreochromis niloticus)
}

\author{
Diego F. Tirado*, Diofanor Acevedo y Luis E. Guzmán \\ Universidad de Cartagena, Facultad de Ingeniería, Departamento de ingeniería de Alimentos, Avenida el \\ Consulado, Calle 30 No. 48-152. Cartagena, Bolívar-Colombia (e-mail: dtiradoa@unicartagena.edu.co) \\ *autor a quien debe ser dirigida la correspondencia
}

Recibido May. 17, 2013; Aceptado Jun. 28, 2013; Versión final recibida Jul. 11, 2013

\begin{abstract}
Resumen
Se determinaron los coeficientes convectivos de transferencia de calor durante el freído por inmersión de láminas de tilapia Oreochromis niloticus en aceite de palma a diferentes temperaturas. Se cortaron filetes de tilapia en forma de placa y sumergieron en aceite a 130,150 , y $170{ }^{\circ} \mathrm{C}$ en una freidora eléctrica de acero inoxidable de seis litros. Las propiedades termofísicas se midieron en función de la temperatura y composición del alimento, usando el programa computacional DEPROTER. Los coeficientes convectivos fueron determinados a partir de gráficas de relaciones adimensionales tiempo -vs- temperatura, y estos disminuyeron al aumentar la temperatura del aceite, presentando valores de $46.76,34.61$ y $32.66 \mathrm{~W} / \mathrm{m}^{2} \mathrm{~K}$ a 130,150 y $170{ }^{\circ} \mathrm{C}$ respectivamente. Se observa que a mayor temperatura del medio de fritura, mayor es la tasa de pérdida de agua desde el producto, y menor su energía interna, y por tanto menor es el coeficiente convectivo de transferencia de calor.
\end{abstract}

\section{Determination of Convective Coefficients of Heat Transfer During Frying of Sheet Tilapia (Oreochromis niloticus)}

\begin{abstract}
The convective heat transfer coefficients during deep-fat frying of Oreochromis niloticus fillets in palm oil at different temperatures were determined. Tilapia fillets were plate-shaped cut and dipped in oil at 130, 150, and $170{ }^{\circ} \mathrm{C}$ in an electric fryer six liter stainless steel. Thermophysical properties were measured as a function of temperature and composition of the food, using the computer program DEPROTER. The convective coefficients were determined from plots of time -vs- dimensionless temperature relationships, and these decreased with increasing oil temperature, with values of $46.76,34.61$ and $32.66 \mathrm{~W} / \mathrm{m}^{2} \mathrm{~K}$ at 130 , 150 and $170{ }^{\circ} \mathrm{C}$ respectively. It was observed that the higher is the temperature of the frying medium, the greater is the rate of water loss from the product, and lower is the internal energy, and therefore lower is the convective heat transfer coefficient.
\end{abstract}

Keywords: heat transfer convective coefficient, immersion frying, tilapia, thermophysical properties 


\section{INTRODUCCIÓN}

Tilapia, nombre común aplicado a tres géneros de peces de la familia Cichlidae: Oreochromis, Sarotherodon y Tilapia. Las especies de mayor importancia económica para la acuicultura son del género Oreochromis (Watanabe et al., 2002) que incluye la tilapia del Nilo (O. niloticus) y tilapia Mozambique (O. mossambicus), entre otras. A nivel mundial, las tilapias son el tercer grupo de peces de mayor importancia, después de la carpa y el salmón, y su cultivo también es una de las actividades de mayor crecimiento (FAO, 2010). Freír por inmersión, puede ser definido como el proceso de cocción de alimentos por inmersión en un aceite o grasa comestible que está a una temperatura superior al punto de ebullición del agua, por lo general 150 a $200{ }^{\circ} \mathrm{C}$ (Lalam et al., 2013; Tirado et al., 2012; Mir-Bel et al., 2012). En esencia, es un proceso de deshidratación rápida (Wu et al., 2013b), en el que contribuyen factores como la temperatura, tiempo de calentamiento, tipo de aceite y el tamaño y naturaleza del producto (Mir-Bel et al., 2012).

Freír los alimentos es un proceso ampliamente usado e industrialmente importante, siendo así un campo de gran interés para los investigadores y científicos de ingeniería, así como de diseñadores, desarrolladores y fabricantes (Wu et al., 2013a). A pesar de esto, sus investigaciones en ingeniería son limitadas, y los snacks como las patatas fritas, son los productos más estudiados, siendo la información acerca de los fenómenos de transporte en los productos de carne frita escasa (Sosa-Morales et al., 2006). El freido se utiliza ampliamente en los sectores domésticos e industriales debido a su capacidad para crear características sensoriales únicas en los alimentos (Ahmad et al., 2013; Aladedunye y Przybylski, 2013). Es un proceso común en la fabricación de alimentos y uno de los más antiguos. En los últimos años se han desarrollado una amplia gama de productos alimenticios fritos, que incluyen alimentos como el pollo y los productos pesqueros, donas, papas fritas, y una variedad de productos snak de rápida expansión, como patatas fritas y otros productos a base de maíz, arroz y trigo (Wu et al., 2013b).

El conocimiento exacto del coeficiente de transferencia de calor durante la fritura es importante en el modelado y cálculo de procesos y equipos, y debe ser cuidadosamente evaluado cuando se quiere preservar la calidad del alimento frito (Mir-Bel et al., 2012). Este valor define en gran medida el tamaño del equipo y establece los contactos entre fases y tiempos de procesamiento (Alvis et al., 2009). Sin embargo, los métodos reportados en la literatura para medir dicho coeficiente presentan algunas limitaciones (Alvis et al., 2009; Moreira et al., 1999) tales como: a) falta de técnicas estandarizadas de medida, b) pocas veces se toma en consideración la transferencia de masa, c) escaso conocimiento del coeficiente de transferencia de calor (en función de la posición del material), d) falta de uniformidad en la estimación y reporte de errores de medida y e) geometría del producto. El presente trabajo tuvo como objetivo determinar los coeficientes convectivos de transferencia de calor durante el freído por inmersión de láminas de tilapia "O. niloticus" en aceite de palma a diferentes temperaturas.

\section{MATERIALES Y MÉTODOS}

Para la determinación del coeficiente convectivo de transferencia de calor se utilizaron filetes de tilapia en forma de placa plana de $5 \times 2,5 \times 1,5 \mathrm{~cm}$. Las condiciones de proceso fueron temperaturas de fritura de 130 , 150 y $170 \stackrel{\circ}{\circ}$ y tiempos de freído entre 1 a $420 \mathrm{~s}$. En este proceso, se empleó aceite de palma como medio de fritura y una freidora eléctrica en acero inoxidable de 6 litros de capacidad. En cada condición de freído se usó una lámina de tilapia y se realizaron tres réplicas analíticas. Para medir la variación de temperatura de las láminas se insertaron 2 termopares tipo $\mathrm{J}$ de $0,25 \mathrm{~mm}$ de diámetro por los extremos de la placa en sentido longitudinal hasta que las puntas de los mismos estuvieron cerca del centro geométrico y suficientemente lejos de los extremos; otro termopar de las mismas características se usó para medir la temperatura del aceite. Las temperaturas se registraron cada segundo empleando un sistema de adquisición de datos (INTECH Micro 2100-A16 Rev 1.3) acoplado a un computador personal. El calor específico y la densidad de la muestra fueron determinados empleando las fórmulas sugerida por Choi y Okos (1985), con base en la composición de la tilapia y la temperatura de fritura. Las propiedades termofísicas de la tilapia se determinaron usando un programa computacional denominado DEPROTER (Determinación de Propiedades Termofísicas) de Alvis et al., (2012) que facilita la determinación del calor específico y la densidad.

El modelo elegido para describir la transferencia de calor durante el freido de láminas de tilapia (Análisis de parámetros concentrados) fue seleccionado por su sencillez frente a otros modelos, como las diferencias finitas o elementos finitos. Este sencillo enfoque ha sido utilizado con los mismos objetivos en otros estudios tales como en el de Moreira et al., (1995b) para fritura de carne de cerdo y Moreira et al., (1995a) en fritura de chips de tortilla. En ambos trabajos el mecanismo de convección es el control de la transferencia de calor. Este modelo se expresa por la ley de enfriamiento de Newton como se presenta en la Ecuación (1):

$Q=h A \Delta T$ 
No obstante, el coeficiente convectivo de transferencia de calor (h) fue calculado para el freído de la tilapia teniendo en cuenta el estado de cuasi equilibrio entre la temperatura del interior de la muestra y el balance de calor, empleando la Ecuación (2), descrita a continuación:

$h A T s-T b=\rho V C p \frac{\partial T}{\partial t}$

Que resolviéndola con las condiciones iniciales y linealizándola, queda expresada como se indica en la Ecuación (3):

$\operatorname{Ln} \frac{\mathrm{Ts}-\mathrm{Te}}{\mathrm{To}-\mathrm{Te}}=\frac{-\mathrm{hA}}{\rho \mathrm{VCp}} \mathrm{t}$

Finalmente, de la pendiente resultante de la gráfica $L n \frac{T s-T e}{T o-T e} V s$. tiempo ( $t$ ) y conocidos el área de trasferencia de calor, la densidad, el calor específico y el volumen de la lámina de tilapia, se determinó $h$ mediante la Ecuación (4) así:

$h=\frac{m \rho C p}{A}$

\section{ANALISIS DE RESULTADOS}

En la Figura 1 se muestra la variación de la temperatura de las láminas de tilapia con el tiempo para diferentes temperaturas de freído. Se observa que en el interior del producto la temperatura aumentó rápidamente al comienzo del proceso, estabilizándose en $85,74^{\circ} \mathrm{C}, 85,74{ }^{\circ} \mathrm{C}$ y $86^{\circ} \mathrm{C}$ a partir de 392,380 y 359 segundos respectivamente, dependiendo de la temperatura del aceite (130, 150 y $170 \stackrel{\circ}{\circ})$. Además, los tiempos de estabilización disminuyeron a medida que aumentaron las temperaturas de proceso, y esto se atribuye a que el interior de la lámina aún no estaba lo suficientemente deshidratado para permitir el incremento de la energía interna (Yildiz et al., 2007).

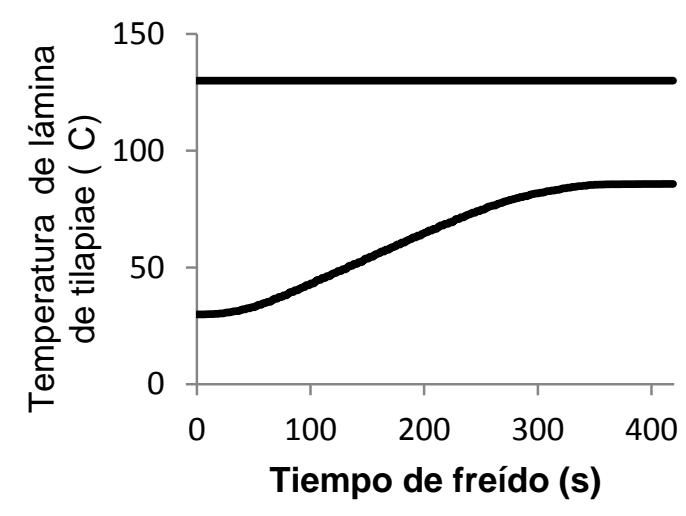

a)

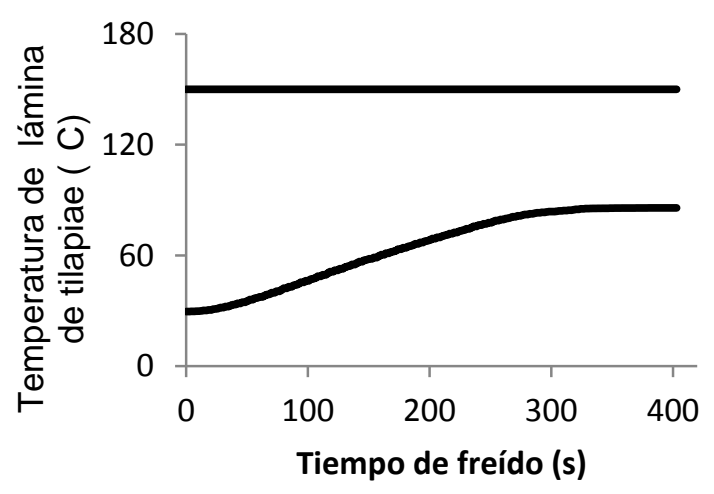

b)

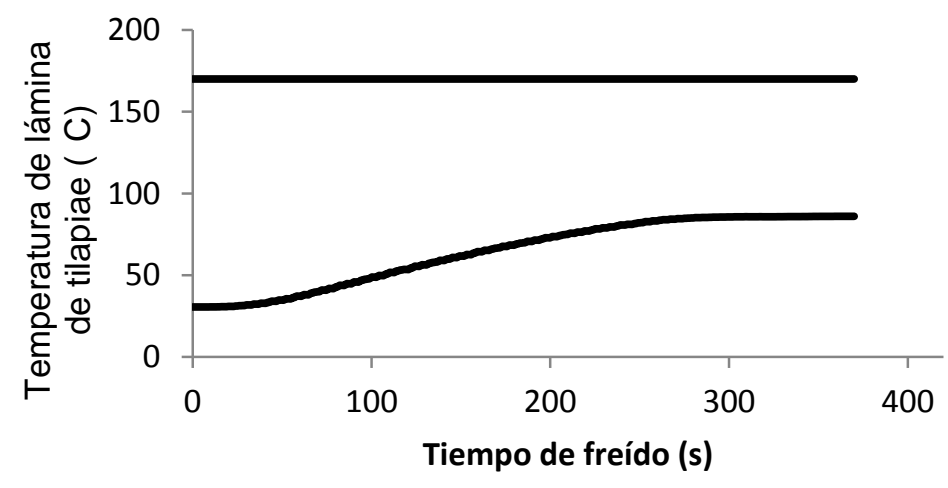

c)

Fig.1: Temperatura de lámina de tilapia durante el freído por inmersión a la temperatura del aceite, a) 130 , b) 150 y c) $170^{\circ} \mathrm{C}$. 
Las pendientes de la sección lineal de la relación adimensional tiempo Vs. temperatura de las curvas, que se obtuvieron mediante análisis de regresión lineal, fueron usadas posteriormente para determinar el coeficiente de transferencia de calor efectivo. En ella se observa que la velocidad de calentamiento de las láminas de tilapia decrece con el incremento de la temperatura de freído.

Tabla 1: Pendientes y coeficientes convectivos de transferencia de calor en función de la temperatura del aceite.

\begin{tabular}{|l|l|l|l|}
\hline Temperatura $\left({ }^{\circ} \mathrm{C}\right)$ & Pendiente $(\mathrm{m})$ & $\mathrm{R}^{2}$ & $\mathrm{~h}\left(\mathrm{~W} / \mathrm{m}^{2} \mathrm{~K}\right)$ \\
\hline 130 & 0,0032 & 0,998 & 46,762 \\
\hline 150 & 0,0024 & 0,999 & 34,617 \\
\hline 170 & 0,0023 & 0,999 & 32,665 \\
\hline
\end{tabular}

En la Tabla 1 se muestran los valores calculados del coeficiente convectivo de transferencia de calor (h) con sus correspondientes pendientes, y se observa que estos disminuyeron al aumentar la temperatura del aceite, presentando valores de $46,76,34,61$ y $32,66 \mathrm{~W} / \mathrm{m}^{2} \mathrm{~K}$ a temperaturas de 130,150 y $170{ }^{\circ} \mathrm{C}$ respectivamente. Estos datos concuerdan con los reportados por Yildiz et al., (2007), quienes encontraron una disminución de los coeficientes convectivos de transferencia de calor con el aumento de la temperatura del aceite de fritura, reportando valores de 286,7, 227,3 y 181,3 W/m $\mathrm{m}^{2} \mathrm{~K}$ para temperaturas de 150,170 y $190 \stackrel{\circ}{\circ}$ respectivamente. Estos autores, junto con Claeys et al., (2005) atribuyen la disminución del coeficiente de transferencia de calor con el aumento de temperatura del aceite a que como el medio de fritura está a una temperatura mayor, esto ocasiona una pérdida de agua desde el producto a una mayor tasa. Cuanto mayor es la tasa de pérdida de agua, mayor será la cantidad extraída de la energía entrante, y esto reduce la cantidad de energía disponible para el aumento de la energía interna, y como resultado el coeficiente de transferencia de calor disminuye. Por otro lado Costa y Oliveira (1999) informan que, aunque el movimiento de las burbujas durante la fritura aumenta la tasa de transferencia de calor, los niveles máximos de las tasas de pérdida de agua pueden obstaculizar la transferencia de calor, y consigo el coeficiente de transferencia de calor.

Los valores de $h$ calculados para la tilapia, se encuentran fuera del rango de valores reportados por algunos autores que trabajaron con productos cárnicos y no cárnicos. Sosa-Morales et al., (2006) ensayaron con placas de carne de cerdo de $5 \times 4 \times 2 \mathrm{~cm}$ en aceite de girasol a 90,100 y $110^{\circ} \mathrm{C}$ y encontraron coeficientes de transferencia de calor de $74,38 \pm 18,57,193,45 \pm 10,87$ y $226,10 \pm 14,29 \mathrm{~W} / \mathrm{m}^{2} \mathrm{~K}$. Por su parte, Califano y Calvelo (1991) reportaron valores de $h$ entre 150 y $165 \mathrm{~W} / \mathrm{m}^{2} \mathrm{~K}$ en el proceso de freído de papas a temperaturas entre 50 y $100{ }^{\circ} \mathrm{C}$; Costa y Oliveira (1999) encontraron valores de $h$ de $594 \pm 38$ y $750 \pm 59$ $\mathrm{W} / \mathrm{m}^{2} \mathrm{~K}$, para trozos de papa en forma de paralelepípedo freídos en aceite de girasol a temperaturas de 140 y $180^{\circ} \mathrm{C}$ por el método indirecto y valores de $443 \pm 32$ y $650 \pm 7 \mathrm{~W} / \mathrm{m}^{2} \mathrm{~K}$ a las mismas temperaturas, por el método directo. Farkas y Hubbard (2000) usaron cilindros de papa para determinar el coeficiente de transferencia de calor durante el freído por inmersión a $180^{\circ} \mathrm{C}$ y encontraron que para un tiempo de 200 a 900 segundos en la fase de ebullición, el valor de $h$ estaba entre 300 y $1100 \mathrm{~W} / \mathrm{m}^{2} \mathrm{~K}$; Budzaki y Seruga (2005), encontraron valores de $h$ entre $94,22 \pm 0,33$ y $774,88 \pm 3,89 \mathrm{~W} / \mathrm{m}^{2} \mathrm{~K}$ para esferas de una mezcla de harina de trigo y papa (50:50) al freírlas en aceite de soya a $190{ }^{\circ} \mathrm{C}$ durante 5 a $120 \mathrm{~s}$, estos autores muestran que los valores más altos del coeficiente de transferencia de calor se observan en la etapa de ebullición y los valores mínimos, en la etapa de velocidad decreciente. Farinu y Baik (2007) trabajaron con esferas de batata sometidas a temperaturas de freído de 150,160,170 y $180^{\circ} \mathrm{C}$ y encontraron valores de $h$ entre 710 y $837 \mathrm{~W} / \mathrm{m}^{2} \mathrm{~K}$.

Las diferentes condiciones y productos utilizados en la determinación de $h$ durante la fritura hacen que sea muy difícil llegar a conclusiones generales válidas para todos los casos de fritura. Por ejemplo, la mayoría de los trabajos publicados han encontrado que $h$ aumenta con la temperatura, mientras que otros han llegado a la conclusión de que disminuye. Esta situación podría estar relacionada con los diferentes patrones de burbujeo de vapor desarrollados durante cada proceso de fritura (Mir-Bel et al., 2012; Alvis et al., 2009).

Los resultados de los coeficientes convectivos de transferencia de calor calculados en esta investigación también son opuestos a los obtenidos por Alvis et al., 2009 y Sahin et al., (1999), quienes trabajaron con ñame y papa, y reportaron un incremento en el coeficiente de transferencia de calor por convección con el aumento de la temperatura de freído. Esto debido a los pequeños poros formados en la costra de los tubérculos durante la fritura por ser ricos en almidón. Por su parte, otras matrices alimenticias como la tilapia, por ser ricas en proteína presentan poros más grande en su costra durante el freído, que ocasionan menos resistencia al vapor de agua, generando un mayor burbujeo durante el proceso de fritura (Bravo, 2008), y en ocasiones la alta velocidad de pérdida de agua genera movimientos vigorosos de las burbujas 
en la interfase sólido/líquido, causando un efecto de barrera, de tal manera que la superficie sólida es prácticamente inaccesible para el aceite, a partir de lo cual, el flujo de calor disminuye, y por ende el coeficiente de transferencia de calor (Mir-Bel et al., 2012; Costa y Olivera, 1999).

\section{CONCLUSIONES}

El coeficiente convectivo de transferencia de calor (h), disminuyó al aumentarse la temperatura de freído. Los valores del coeficiente de transferencia de calor fueron 46,76, 34,61 y $32,66 \mathrm{~W} / \mathrm{m}^{2} \mathrm{~K}$, a las temperaturas de 130,150 y $170{ }^{\circ} \mathrm{C}$. A mayor temperatura del medio de fritura, mayor es la tasa de pérdida de agua desde el producto y menor es su energía interna, por tanto menor es el coeficiente convectivo de transferencia de calor. Por otro lado, la relación conjunta entre transferencia de calor, masa y burbujeo, hace el freír un proceso difícil, provocando movimientos vigorosos de las burbujas en la interfase sólido/líquido, que disminuyen el coeficiente convectivo de transferencia de calor.

\section{NOTACIÓN}

$\begin{array}{ll}Q & \text { Velocidad de flujo de calor }(\mathrm{J} / \mathrm{s}) \\ h & \text { Coeficiente de transferencia de calor }\left(\mathrm{W} / \mathrm{m}^{2} \mathrm{~K}\right) \\ \Delta T & \text { Gradiente de temperatura }\left({ }^{\circ} \mathrm{C}\right) \\ A & \text { Área de superficie }\left(\mathrm{m}^{2}\right) \\ t & \text { Tiempo }(\mathrm{s}) \\ T b & \text { Temperatura del medio de freído }\left({ }^{\circ} \mathrm{C}\right) \\ T s & \text { Temperatura localizada en un punto a un tiempo } \mathrm{t}\left({ }^{\circ} \mathrm{C}\right) \\ \rho & \text { Densidad }\left(\mathrm{Kg} / \mathrm{m}^{3}\right) \\ C p & \text { Calor especifico }\left(\mathrm{kJ}^{\mathrm{N}} / \mathrm{kg}^{\circ} \mathrm{C}\right) \\ V & \text { Volumen de la lámina de tilapia }\left(\mathrm{m}^{3}\right) \\ T_{0} & \text { Temperatura de producto al inicio del proceso }\left({ }^{\circ} \mathrm{C}\right) \\ m & \text { Pendiente de la sección lineal. }\end{array}$

\section{REFERENCIAS}

Ahmad, A., K. Niranjan, y M. Gordon, Physico-chemical changes occurring in oil when atmospheric frying is combined with post-frying vacuum application, Food Chemistry: 136(2), 902-908 (2013).

Aladedunye, F. y R. Przybylski, Frying stability of high oleic sunflower oils as affected by composition of tocopherol isomers and linoleic acid content, Food Chemistry: 141(3), 2373-2378 (2013).

Alvis, A., I. Caicedo y P. Peña, Determinación de Propiedades Termofísicas de Alimentos en Función de la Concentración y la Temperatura empleando un Programa Computacional, Información Tecnológica, 23(1), $111-116$ (2012).

Alvis, A., L. Cortés y M. Páez, Transferencia de Calor y Materia durante la Fritura de Trozos de Ñame (Dioscórea alata), Información Tecnológica: 20(1), 99-109 (2009).

Bravo, J., Contribución al estudio de la fritura al vacío: Deshidratación de rodajas de manzana, Tesis Doctoral, Departamento de Tecnología de Alimentos, Universidad Politécnica de Valencia, Valencia España (2008).

Budzaki, S. B. y Seruga, Determination of convective heat transfer coefficient during frying of potato dough, Journal Food Engineering: 66(3), 307-314 (2005).

Califano, A.N. y A. Calvelo, Thermal conductivity of potato between 50 and $100^{\circ} \mathrm{C}$, Journal of Food Science: 56, 586-589 (1991).

Choi, Y. y M.R. Okos, Thermal properties in liquid foods-Review. In: Physical and Chemical properties of food. Ed. M.R. Okos, American Society of Agricultural Engineering, p.p. 93-101 (1985).

Claeys, W. L., K. De Vleeschouwer y M.E. Henrickx, Quantifying the formation of carcinogens during food processing: Acrylamide, Trends in Food Science and Technology: 16(5), 181-193 (2005). 
Costa, R.M. y F.A. Oliveira, Modelling the kinetics of water loss during potato frying with a compartmental dynamic model, Journal of Food Engineering: 41(3-4), 177-185 (1999).

FAO (Food and Agriculture Organization of the United Nations), El estado mundial de la Pesca y la acuicultura (2010), http://www.fao.org/docrep/013/i1820s/i1820s.pdf. Acceso: 15 Octubre (2012).

Farinu, A. y O.D. Baik, Heat transfer coefficients during deep fat frying of sweet potato: Effects of product size and oil temperature, Food Research International: 40(8), 989-994 (2007).

Farkas, B. E. y L.J. Hubbard, Analysis of convective heat transfer during immersion frying drying, Journal of Food Technology: 18(5), 145 -151 (2000).

Lalam, S. y otros cuatro autores, Experimental study on transport mechanisms during deep fat frying of chicken nuggets, LWT-Food Science and Technology: 50(1), 110-119 (2013).

Mir-Bel, J., R. Oria y M. Salvador, Influence of temperature on heat transfer coefficient during moderate vacuum deep-fat frying, Journal of Food Engineering: 113(2), 167-176 (2012).

Moreira, R.G., M.E. Castell-Perez y M.A. Barrufet, Deep-fat frying. Fundamentals and applications, An aspen publication, Gaithersburg, Maryland, pp. 75-177 (1999).

Moreira, R. G., J. Palau y X. Sun, Simultaneous heat and mass transfer during deep fat frying of tortilla chips, Journal of Food Process Engineering: 18(3), 307-320 (1995a).

Moreira, R. y otros tres autores, Thermal and physical properties of tortilla chips as a function of frying time, Journal of Food Processing and Preservation: 19(4), 175-189 (1995b).

Sahin, S., S.K. Sastry, y L. Bayindirli, Heat transfer during frying of potato slices, Journal of Food Engineering: 32(1), 19-24 (1999).

Sosa-Morales, M.E., R. Orzuna-Espiritu y J.F. Velez-Ruiz, Mass, thermal and quality aspects of deep-fat frying of pork meat, Journal of Food Engineering: 77(3), 731-738 (2006).

Tirado, D.F., D. Acevedo y L.E. Guzmán, Freído por inmersión de los alimentos, Revista Reciteia: 12(1), 6982 (2012).

Watanabe, W.O. y otros tres autores, Tilapia Production Systems in the Americas: Technological Advances, Trends, and Challenges, Fisheries Science: 10(3-4), 465-498 (2002).

Wu, H., T.G. Karayiannis y S.A. Tassou, A two-dimensional frying model for the investigation and optimisation of continuous industrial frying systems, Applied Thermal Engineering: 51(1-2), 926-936 (2013a).

$\mathrm{Wu}, \mathrm{H}$. y otros tres autores, Analysis and simulation of continuous food frying processes, Applied Thermal Engineering: 53(2), 332-339 (2013b).

Yildiz, A.T., K. Palazoglu y F. Erdogdu, Determination of heat and mass transfer parameters during frying of potato slices, Journal of Food Engineering: 79(1), 11-17 (2007). 